\title{
A Simple Model of a Water/Fuel Spray Diffusion Flame
}

\author{
Jerrold Barry Greenberg*, Yonit Mindelis** \\ Faculty of Aerospace Engineering \\ Technion - Israel Institute of Technology \\ Haifa 32000, Israel \\ *corresponding author: aer9801@technion.ac.il, **yonit.mindelis@gmail.com
}

\author{
Richard L. Axelbaum*** \\ Department of Energy, Environmental \& Chemical Engineering, \\ Consortium for Clean Coal Utilization, \\ Washington University in St. Louis, St. Louis 63130, USA \\ ***axelbaum@wustl.edu
}

\begin{abstract}
The classical Burke-Schumann diffusion flame is adopted as a paradigm configuration for examining spray diffusion flames in which the spray's droplets are comprised of a mixture of water and a miscible fuel. Building on experimental data and simple modeling predictions from the literature, which testify to the viability of burning fuels heavily diluted with water, a special fuel vaporization Damköhler number was constructed to reflect preferential fuel evaporation and non-ideal solution behavior of the spray's water/fuel droplets. An analytical solution of the governing equations was found. Calculations based on the solution enabled a comparison between water/tbutanol and water/ethylene-glycol spray diffusion flames. The former spray was characterized by strong enhanced fuel evaporation whereas the latter by weak enhanced evaporation. Noticeable differences in flame shapes, heights and temperatures were found for the two spray flames. It is argued that the possibility exists of sustaining water/fuel spray diffusion flames by combining the enhanced fuel evaporation and control of the oxygen supply.
\end{abstract}

Key words: Diffusion flame, high water content fuel, preferential vaporization, spray combustion, water/fuel droplets 


\section{Introduction}

The viability of burning fuels heavily diluted with water has been demonstrated by Yi [1] and Yi and Axelbaum [2,3]. The motivation for these studies was the examination of an approach to extract chemical energy by direct combustion from fuels that naturally contain high water content. In contrast to common methods, the suggested approach avoids the energy-demanding process of water removal, thereby increasing the overall energy efficiency. Also, if used in pressurized systems, the latent heat used to evaporate the water can be recovered and utilized. In addition, if oxygen is used as the oxidizer, it allows for efficient carbon capture. Recently, it was reported that utilization of direct steam generation with a natural gas burner to generate power, while enabling carbon capture, is being carried out commercially [4].

Although the combustion of mixtures of water and fuel had been studied in the literature (see references in $[2,3]$ ) the novel features revealed by Yi and Axelbaum's experiments (backed up by some theoretical support through predictions of an elementary theory of single droplet evaporation/distillation) were that preferential evaporation of, for example, ethanol over water plays a key role in enhancing flame stability in the swirl-stabilized burner configuration, and that non-ideal solution behavior should be accounted for when modeling the evaporation process. Preferential evaporation occurs when one chemical substance of a droplet's mixture evaporates more rapidly than another component. In a simple sense, a comparison of the evaporation of pure droplets of two different substances would reveal that one droplet would evaporate more rapidly than the other due to its greater volatility. But, in addition, the greater rate of evaporation may be further enhanced. Generally, in an ideal mixture, the molecular interactions between each pair of chemical species are the same. However, in non-ideal mixtures, molecules of the different components may 
interact to repel each other. This non-ideal state is expressed through the activity coefficient, which identifies the actual partial pressure of the substance relative to its partial pressure if it were an ideal liquid. Importantly, the activity coefficient is a function of the composition of droplet and in water/fuel mixtures it can be quite large (>3) when the concentration of the fuel is small, particularly for alcohols. To a lesser or greater extent both the aforementioned mechanisms were found to play a role in the water/fuel spray flames considered.

The complex behavior of evaporating (and combusting) multicomponent droplets has been recognized for some time. Newbold and Amundson [5] proposed a model for the evaporation of a multicomponent droplet. Law [6] gave a theory of internal boiling and superheating of vaporizing multicomponent droplets in a quiescent atmosphere and analyzed the different stages of development within the evaporating droplet (see also [7]). An extensive historical overview of, and new advanced developments in this area appear in Sazhin [8].

In a related theoretical publication Hou et al. [9] presented a mathematical analysis of the interaction between internal heat gain and loss on compound drop spray flames. In these compound droplets a water core is surrounded by $n$-octane fuel. Evaporation occurs sequentially as the water does not begin to evaporate until the "outer shell" of fuel has completely evaporated. For a premixed compound drop spray flame the influence on the burning velocity of the competing heat gain, due to fuel vapor made available for combustion, and heat loss, due to water evaporation, was mapped out in terms of the relative amounts of fuel and water in the droplets.

However, it is well-known that the behavior of a spray of droplets is not always reflected through that of a single droplet multiplied by the number of droplets in the spray. In the current work we consider the aforementioned findings of $\mathrm{Yi}$ and 
Axelbaum, viz. preferential fuel evaporation and non-ideal solution behavior, in a phenomenological manner within the simple modeling context of the classical gas laminar Burke-Schumann diffusion flame [10] subsequently extended in [11, 12] to the case of a fuel spray diffusion flame. Here, we investigate a Burke-Schumann type of diffusion flame fired by a water/fuel spray of well-stirred liquid droplets. Although devoid of the intricate details afforded by a more all-encompassing numerical model, experience has shown that relatively simple models of this nature, which enable a mathematical analysis, can serve two main functions: (a) they provide convenient benchmarks for full computational solutions of the problem, and (b) they offer clear, uncluttered insight into the physico-chemical underpinnings of spray-gas interaction and how it impacts combustion. It is from this viewpoint that the current investigation is carried out.

\section{Mathematical Model - Governing Equations}

The Burke-Schumann spray flame configuration (see Figure 1) is considered, in which fuel vapor and water/fuel droplets flow in an inner duct (of half width $L$ ) and oxygen flows in outer ducts (each of width $R-L$ ). Under appropriate operating conditions, after diffusive mixing of the two streams, a laminar, spray diffusion flame is established. The velocities of the streams in the inner and outer ducts are taken to be identical and constant, $(u, v)=(0, v)$. A recent sophisticated approach to modeling monodisperse spray combustion is given in $[13,14]$, in which there appears a description of the way in which the different scales relevant to the droplets and the host gas can be thoroughly exploited to formulate spray combustion in both the finite and infinite chemical-Damköhler limit. Advantages of the formulation are the ability to account for non-unity Lewis numbers for the gas phase and heat, mass and momentum transfer between the liquid and gas phases in terms of physically-meaningful 
mixture fractions and excess enthalpies. However, despite its proven advantages and the insight it affords the approach has yet to be applied to polydisperse and multicomponent spray combustion.

In the current work, the description of the spray that is adopted is based on the alternative proven sectional approach [15] (see also, for example, $[11,12,16]$ ). In this approach the pointwise size distribution of droplets in the spray is subdivided into a finite number of size sections each of which contains droplets that fall within a certain size range. The mass balance in any section $\mathrm{j}$ accounts for (a) influx of droplets from the next section up, $\mathrm{j}+1$, which have diminished in size and have thus become eligible for membership in section $\mathrm{j}$, and (b) convection and mass loss due to evaporation of droplets in section $\mathrm{j}$. Here, for simplicity, we take the spray to be mono-sectional, that is, we assume that a single sectional equation is sufficient to describe the spray's characteristics. Use of a single integral quantity (here the mass fraction of the liquid comprising the droplets in the spray) does not negate redistribution of the range of droplet sizes under consideration. However, it does mean that only overall properties of the spray and its effects can be accounted for. In the current context this approach is acceptable. To focus on the essential features of interest it is assumed that the droplets are located in the far-field in relation to the spray source [17] so that they have settled into dynamic and thermal equilibrium with their host gas carrier. In addition, the Lewis number is assumed to be unity. This is generally not too restrictive, but does provide a basis for an analysis for cases of more realistic nonunity Lewis numbers. Also, it is emphasized that changes in the basic flame characteristics (e.g. flame shape \&c.) are not qualitatively effected by Lewis number. The governing equations then assume the following form. For the spray: 
$v \frac{\partial m_{d, F}}{\partial y}=-\tilde{C}_{F} m_{d, F} H(L-x)$

$v \frac{\partial m_{d, W}}{\partial y}=-\tilde{C}_{W} m_{d, W} H(L-x)$

in which $m_{d, F}$ and $m_{d, W}$ are the mass fractions of liquid fuel and water, respectively, in the sprays droplets and $\tilde{C}_{F}$ and $\tilde{C}_{W}$ are their sectional vaporization coefficients. The (non-dimensional) form of these vaporization coefficients will be discussed later. Suffice to say now, they contain within them information about such properties as the evaporation properties of the component liquid and the range of droplet sizes under consideration [15]. Coupled to these equations are the conservation equations for the Schwab-Zeldovitch parameters $m$ and $m_{T}$ which are derived from the conservation equations for fuel vapor, oxygen and energy

$$
\begin{aligned}
& v \frac{\partial m}{\partial y}=D_{g}\left[\frac{\partial^{2} m}{\partial y^{2}}\right]+\tilde{C}_{F} m_{d, F} H(L-x) \\
& v \frac{\partial m_{T}}{\partial y}=D_{g}\left[\frac{\partial^{2} m_{T}}{\partial y^{2}}\right]+\left(1-\beta_{F}\right) \tilde{C}_{F} m_{d, F} H(L-x)-\beta_{W} \tilde{C}_{F} m_{d, W} H(L-x)
\end{aligned}
$$

in which $m$ is defined by

$$
m=m_{F}-m_{O} / v
$$

where $m_{F}, m_{O}$ are the fuel vapor and oxygen mass fractions, respectively, and $v$ is the stoichiometric coefficient associated with the single step global chemical reaction that is assumed to describe the chemistry of the diffusion flame, $F+v O \rightarrow$ Products. It is further assumed that the chemical Damköhler number $D a$ is infinitely large so that the flame is limited to a thin surface into which fuel vapor and oxidant flow from opposite sides of the front. This precludes the possibility of examining questions of stability, extinction and unsteadiness which are therefore beyond the scope of this work. Nevertheless, as a starting point, from a far-field viewpoint the flame surface assumption here provides useful information about steady state flame characteristics, as will be shown. In Eqs.(3) and (4) $D_{g}$ is the diffusion coefficient taken to be 


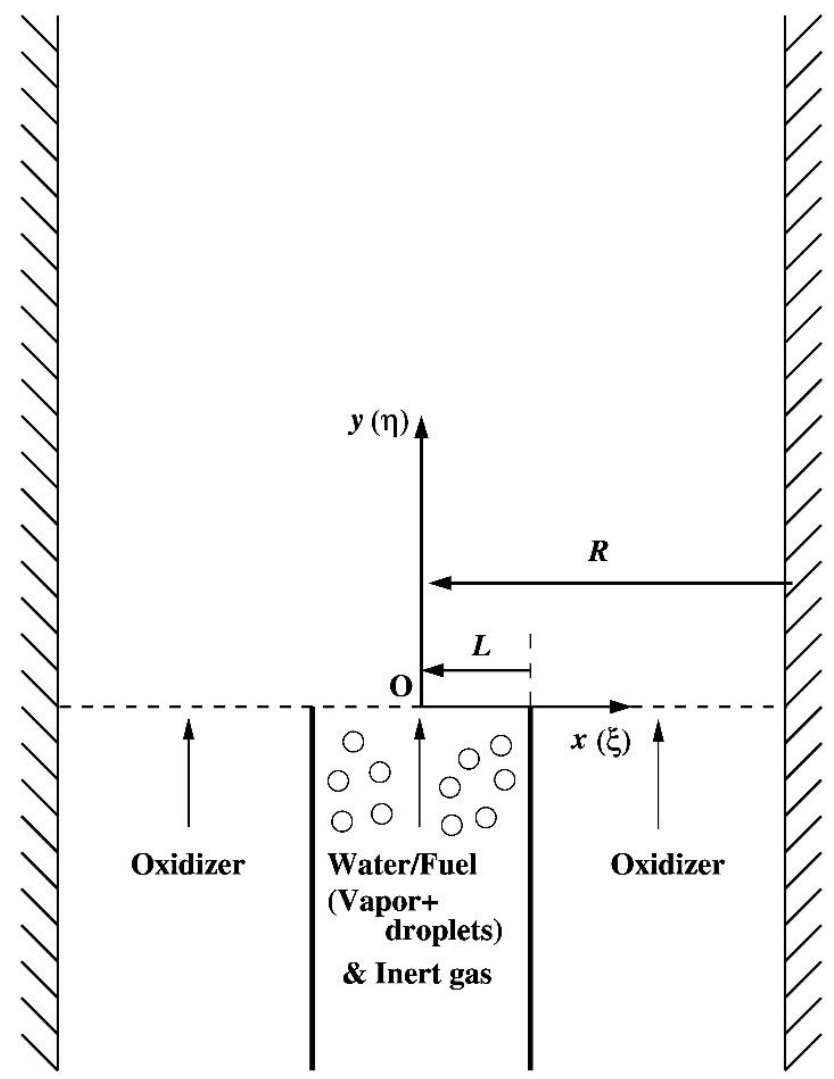

Figure 1: Configuration for formation of Burke-Schumann spray diffusion flame.

identical for the fuel vapor and the oxygen and, as mentioned before, the Lewis number is taken to be unity. In Eq.(4) $m_{T}$ is defined by

$m_{T}=m_{F}+T^{*}$

where $T^{*}$ is the temperature and $\beta_{F}, \beta_{W}$ are the ratios of the latent heat of vaporization of the liquid fuel and water, respectively, to the heat of reaction. Finally, the Heaviside function, $H$, is used to emphasize that the spray related source/sink terms are restricted to the region vertically above the fuel supply duct (see Fig. 1) as in this model there is no mechanism allowing droplets to move laterally.

The boundary conditions for the governing equations are:

$$
\begin{aligned}
& 0 \leq x \leq L, y=0: m_{d, F}=m_{d, F O}, \quad m_{d, W}=m_{d, W O} \\
& m=m_{F}, m_{T}=m_{F}+T_{0} \\
& L \leq x \leq R, y=0: m=-\frac{m_{O O}}{v}, m_{T}=T_{0} \\
& x=0, R, y \geq 0: \frac{\partial m}{\partial x}=\frac{\partial m_{T}}{\partial x}=0
\end{aligned}
$$


Conditions (7a) specify the liquid phase's entrance mass fractions, (7b) the inlet fuel vapor mass fraction and its combination with the temperature, $(7 \mathrm{c})$ the inlet oxygen mass fraction and the temperature and (7d) symmetry (at $x=0$ ) and impermeability of the outer wall of the chamber (at $x=R$ ) to both mass and heat transfer, respectively.

\section{Normalization of the Governing Equations}

In order to finalize the formulation of the governing equations it will be instructive to normalize the various independent and dependent variables. Following [11], use is made of the following

$$
\begin{aligned}
& (\xi, \eta, c)=\left(\frac{x}{R}, \frac{y D_{g}}{v R^{2}}, \frac{L}{R}\right) \\
& \left(\gamma, \gamma_{d, F}, \gamma_{d, W}, V\right)=\left(m, m_{d, F}, m_{d, W}, m_{O O} / v\right) / m_{\text {totfuel }} \\
& \gamma_{T}=\gamma_{F}+T
\end{aligned}
$$

where the non-dimensional temperature is defined by

$T=\left(\left(T^{*}-T_{0}\right) / T_{\text {ref. }}\right)$

in which $T_{r e f}$. is some reference temperature.

In these definitions $m_{\text {totfuel }}$ is the total mass fraction of fuel and water (i.e. vapor+liquid) at the outlet of the inner duct. In addition, the following parameter is defined

$$
\alpha=\frac{m_{d, W, 0}}{m_{d, F, 0}+m_{d, W, 0}}
$$

representing the initial relative liquid-phase mass fraction of water to fuel+water in the droplets.

Applying these definitions leads to the following form for the governing nondimensional equations

$$
\frac{\partial \gamma_{d, F}}{\partial \eta}=-\Delta_{F} \gamma_{d, F} H(c-\xi)
$$




$$
\begin{aligned}
& \frac{\partial \gamma_{d, W}}{\partial \eta}=-\Delta_{W} \gamma_{d, W} H(c-\xi) \\
& \frac{\partial \gamma}{\partial \eta}=\frac{\partial^{2} \gamma}{\partial \xi^{2}}+\Delta_{F} \gamma_{d, F} H(c-\xi) \\
& \frac{\partial \gamma_{T}}{\partial \eta}=\frac{\partial^{2} \gamma_{T}}{\partial \xi^{2}}+\Delta_{F}\left(1-\beta_{F}\right) \gamma_{d, F} H(c-\xi)-\Delta_{W} \beta_{W} \gamma_{d, W} H(c-\xi)
\end{aligned}
$$

subject to boundary conditions:

$$
\begin{gathered}
0 \leq \xi \leq c, \eta=0: \gamma_{d, F}=\delta_{F}=(1-\alpha) \delta, \gamma_{d, W}=\delta_{W}=\alpha \delta \\
\gamma=1-\delta, \gamma_{T}=1-\delta+T_{0} \\
c \leq \xi \leq 1, \eta=0: \gamma=-V, \gamma_{T}=T_{0} \\
\xi=0,1, \eta \geq 0: \frac{\partial \gamma}{\partial \xi}=\frac{\partial \gamma_{T}}{\partial \xi}=0
\end{gathered}
$$

in which $\delta$ is the initial fraction of liquid fuel and water in the total fuel/water supply (which may also include fuel vapor). This is in contradistinction to the definition of $\alpha$ (Eq. (12) which is the initial relative liquid-phase mass fraction of water to fuel+water in the droplets. Finally, the so-called vaporization Damköhler numbers are $\Delta_{j}=\tilde{C}_{j} R^{2} / D_{g}$, with $j=F, W \quad$ (see $[18,19]$ where the terminology "vaporization Damköhler number" is borrowed from the standard chemical kinetics usage).

\section{Modeling Water/Fuel Droplet Phenomena in the Spray}

In the previous section the vaporization Damköhler numbers for the fuel and water content of the droplets were introduced. Generally, it is sufficient to take these parameters as appropriate constants in order to extricate a qualitatively correct description of the interaction of the spray with and its influence on the combustion field (see, for example, $[11,12,16]$. However, to capture the features of water/fuel droplet vaporization as delineated in $[1,2,3]$ a modification is required. Whereas the water vaporization Damköhler number can still be taken as constant a more subtle description is required for the construction of the fuel vaporization Damköhler 
number. It should mirror the finding that the rate of evaporation of the liquid fuels considered could be strongly affected by their activity coefficient. The activity coefficient is a factor used in thermodynamics to account for the deviation from ideal mixture behavior. In an ideal mixture, the microscopic interactions between pairs of chemical species are the same (or macroscopically equivalent, the enthalpy change of solution and volume variation in mixing is zero) and, as a result, properties of the mixtures can be expressed directly in terms of simple concentrations or partial pressures of the substances present e.g. through Raoult's law. Deviations from ideality are accommodated by modifying the concentration by an activity coefficient. In nonideal mixtures, molecules of the different components may interact to repel each other leading to enhanced evaporation of one component over the other. Indeed, this behavior was found in previous calculations $[1,2]$ which showed that when water is present in the droplets the lower the fuel content the greater the rate at which fuel was released as vapor. To model this special feature in the current context the following expression was adopted for the fuel vaporization Damköhler number:

$$
\Delta_{F}=\Delta_{F 0}\left(1+F_{1} a_{c}\right)\left(\frac{1-F_{2} a_{c} \delta}{1-a_{c} \delta}\right)
$$

where $\Delta_{F 0}$ is a nominal constant vaporization Damköhler number relating to droplets composed of liquid fuel only, that can be evaluated in the usual fashion [15], and

$$
\left(F_{1}, F_{2}\right)=\left(A_{c} \varphi,\left(\frac{\gamma_{d, F}}{\gamma_{d, W}}\right) \cdot\left(\frac{\alpha}{1-\alpha}\right)\right)
$$

in which $0 \leq a_{c} \leq 1$ is dubbed a normalized "activity" coefficient, $A_{c}$ is a normalization factor based on [3] and $0 \leq \varphi \leq 1$ is an influence coefficient (all to be explained later). 
First, the role of each expression in brackets in Eq.(21) is described. The first term on the RHS designates the initial factor by which the nominal vaporization coefficient must be increased due to the presence of water in the droplets. The more sensitive a fuel is to being repelled by water on the molecular level the closer the value of $a_{c}$ will be to unity. For convenience, the extent of this increase is artificially controlled through the parameter $\varphi$. The second bracketed term on the RHS describes the way the initial vaporization Damköhler number alters as the ratio between the liquid fuel and the water in the droplets varies during their spatial evolution downstream. At the entrance to the chamber $F_{2}$ is unity. Moving downstream, for fuel evaporating more rapidly than water from the droplets, $F_{2}$ will be less than unity thereby ensuring that the expression in the second brackets of Eq. (21) will be greater than one so that $\Delta_{F}$ will increase accordingly - this being in accordance with the notion that with water present in the droplets the lower the fuel content the greater the rate at which fuel is released as vapor. Finally, the preferential evaporation of fuel over water is exhibited through the ratio $\Delta_{F 0} / \Delta_{W}$. Thus, the structure of the modified fuel vaporization Damköhler number qualitatively captures the features of water/fuel droplets evaporation as featured in $[1,2,3]$

The parameters relating to the activity coefficient were determined by making use of Fig. 2 of [3] in which the activity coefficient is plotted as a function of water content for droplets of several liquid fuel/water combinations. The maximum value of the activity coefficient in the graph, $A_{c}$, was used to create a normalized activity coefficient, $a_{c}$. Thus, if the activity coefficient of [3] is denoted by $\bar{a}_{c}$ the values of 
$a_{c}$ are found from $\bar{a}_{c}=A_{c} a_{c}+1$. In practice, the following function was used to fit the curves for each fuel

$a_{c}\left(\alpha, a_{0}, \psi\right)=-a_{0}\left[\frac{\arctan \left(a_{0} \psi(1-\alpha)\right)}{\arctan \left(a_{0} \psi\right)}-1\right]$

in which $a_{0}$ is the maximum value of $a_{c}$ for a given chemical substance obtained from Fig. 2 of [3] when $\alpha=1$. Table 1 shows the values of $a_{0}$ and $\psi$ giving the best fits for t-butanol and ethlylene glycol which are both water soluble chemicals.

\begin{tabular}{|c|c|c|}
\hline Fuel & $a_{0}$ & $\psi$ \\
\hline t-butanol & $\mathbf{0 . 9 7 5}$ & 8.5 \\
\hline Ethylene glycol & $\mathbf{0 . 0 3 4 5}$ & 120 \\
\hline
\end{tabular}

Table 1: Fitting function parameters for water soluble chemicals based on Fig. 2 of reference [3].

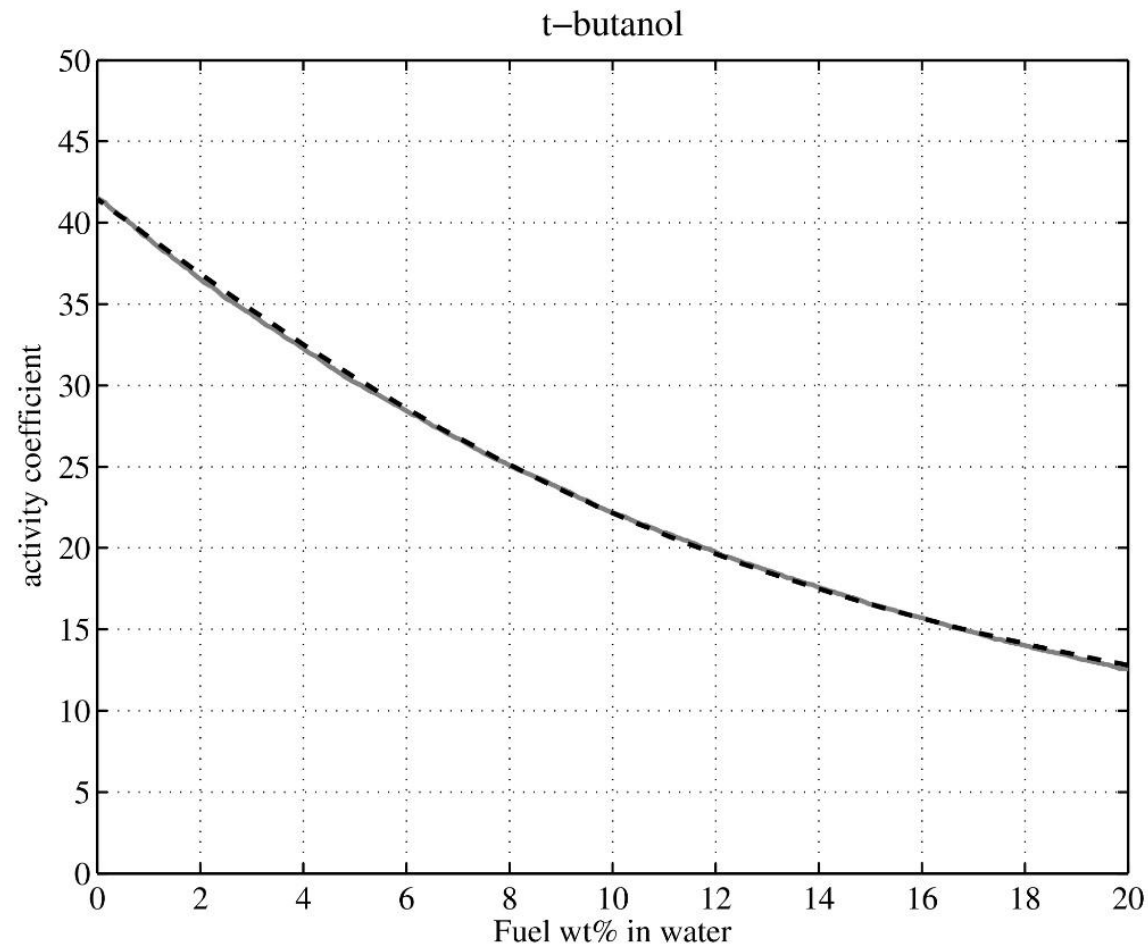

Figure 2a: Activity coefficient vs percentage of fuel in water by weight for $t$ butanol-water droplets; comparison between data fit using Eq.(23) (broken black line) and data of reference [3] (grey line). 


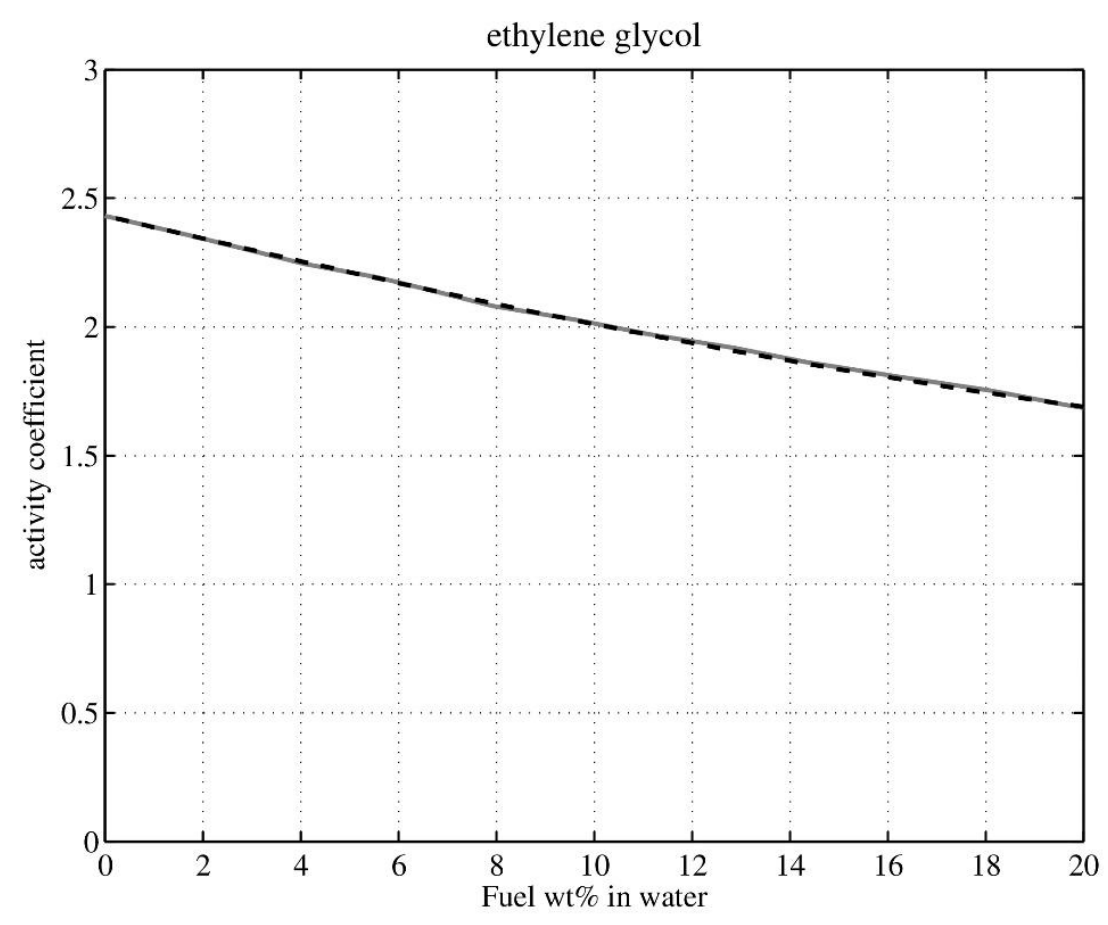

Figure 2b: Activity coefficient vs percentage of fuel in water by weight for ethylene glycol-water droplets; comparison between data fit using Eq.(23) (broken black line) and data of reference [3] (grey line).

Use of Eq. (23) is shown in Fig. 2a for t-butanol and Fig. 2b for ethylene-glycol. The agreement is excellent, as can be seen by the almost perfect overlap of the approximation (broken black line) and the data from [3] (solid grey line).

In view of the afore-described, it is instructive to rewrite the relevant governing equations in full. Eqs (13), (15) and (16) become

$$
\begin{aligned}
& \frac{\partial \gamma_{d, F}}{\partial \eta}=-\hat{\Delta}_{F O}\left(1+A \frac{\gamma_{d, F}}{\gamma_{d, W}}\right) \gamma_{d, F} H(c-\xi) \\
& \frac{\partial \gamma}{\partial \eta}=\frac{\partial^{2} \gamma}{\partial \xi^{2}}+\hat{\Delta}_{F O}\left(1+A \frac{\gamma_{d, F}}{\gamma_{d, W}}\right) \gamma_{d, F} H(c-\xi) \\
& \frac{\partial \gamma_{T}}{\partial \eta}=\frac{\partial^{2} \gamma_{T}}{\partial \xi^{2}}+\left(1-\beta_{F}\right) \hat{\Delta}_{F 0}\left(1+A \frac{\gamma_{d, F}}{\gamma_{d, W}}\right) \gamma_{d, F} H(c-\xi)-\Delta_{W} \beta_{W} \gamma_{d, W} H(c-\xi)
\end{aligned}
$$

in which

$$
\hat{\Delta}_{F 0}=\Delta_{F 0}\left(\frac{1+F_{1} a_{c}}{1-a_{c} \delta}\right)
$$


and

$$
A=-\left(\frac{\alpha}{1-\alpha}\right) a_{c} \delta
$$

The non-linear nature of the spray source terms expresses the required complex interaction resulting from the water/fuel mixture in the droplets.

In summary, the governing equations to be solved are Eqs. (14), (24)-(26) subject to the boundary conditions Eqs. (17)-(20).

\section{Solution for Diffusion Flame}

The solution of Eq. (14) is readily shown to be:

$\gamma_{d, W}=\alpha \delta e^{-4_{w} \eta} H(c-\xi)$

Eq. (24) is a Riccati equation whose solution, after substituting for $\gamma_{d, W}$ from Eq. (29), is

$$
\gamma_{d, F}=\frac{(1-\alpha) \delta e^{-\hat{-}_{F O} \eta}}{1-\left(\frac{\hat{\Delta}_{F 0}}{\hat{\Delta}_{F 0}-\Delta_{W}}\right) a_{c} \delta\left(1-e^{-\left(\hat{\Delta}_{F 0}-\Delta_{W}\right) \eta}\right)} H(c-\xi)
$$

Armed with these solutions for $\gamma_{d, F}$ and $\gamma_{d, W}$, the equations for the SchwabZeldovitch parameters can be tackled. The solution for $\gamma$ is

$$
\begin{aligned}
& \gamma(\xi, \eta)=c[(1-\delta)+V]-V+c \hat{\Delta}_{F 0} I_{0}+ \\
& +\frac{2}{\pi}[(1-\delta)+V] \sum_{n=1}^{\infty} \frac{1}{n} \sin (n \pi c) \cos (n \pi \xi) e^{-(n \pi)^{2} \eta}+ \\
& +\frac{2}{\pi} \sum_{n=1}^{\infty} \frac{1}{n} \sin (n \pi c) \cos (n \pi \xi) \hat{\Delta}_{F 0} e^{-(n \pi)^{2} \eta} I_{n}
\end{aligned}
$$

where

$$
I_{n}=\int_{0}^{\eta} e^{(n \pi)^{2} \eta} \Phi(\eta) d \eta, \text { for } n=0,1,2 \ldots \ldots \ldots
$$

and

$$
\Phi(\eta)=\left(1+A \frac{\gamma_{d, F}}{\gamma_{d, W}}\right) \gamma_{d, F}
$$


The integrals are evaluated numerically using the trapezoidal rule.

In a similar manner the temperature-related $\gamma_{T}$ is found to be

$$
\begin{aligned}
& \gamma_{T}(\xi, \eta)=c(1-\delta)+c\left(1-\beta_{F}\right) \hat{\Delta}_{F 0} I_{0}-c \alpha \beta_{W}\left(1-e^{-\Delta_{W} \eta}\right) \\
& +\frac{2}{\pi}(1-\delta) \sum_{n=1}^{\infty} \frac{1}{n} \sin (n \pi c) \cos (n \pi \xi) e^{-(n \pi)^{2} \eta}+ \\
& +\frac{2}{\pi}\left(1-\beta_{F}\right) \sum_{n=1}^{\infty} \frac{1}{n} \sin (n \pi c) \cos (n \pi \xi) \hat{\Delta}_{F 0} e^{-(n \pi)^{2} \eta} I_{n} \\
& -\frac{2}{\pi} \alpha \delta \beta_{W} \sum_{n=1}^{\infty} \frac{1}{n} \sin (n \pi c) \cos (n \pi \xi) \frac{\Delta_{W}}{\left((n \pi)^{2}-\Delta_{W}\right)}\left(e^{-\Delta_{W} \eta}-e^{-(n \pi)^{2} \eta}\right)
\end{aligned}
$$

Using Eq. (31) the diffusion flame front can be located at the locus of all points $(\xi, \eta)$ for which $\gamma(\xi, \eta)=0$. Given the solution for $\gamma$ (and, hence, $\gamma_{F}$ ) the temperature field can be extracted using Eq. (10).

\section{Results and Discussion}

The basic data set utilized for applying the solutions obtained to investigate the water/fuel spray diffusion flames was as follows, unless otherwise specified: $c=1 / 6, V=0.306, \delta=1, \beta_{F}=0.04, \beta_{W}=0.08, \alpha=0.8, \phi=0.01, \Delta_{F 0} / \Delta_{W}=100$.

In Figure 3 the diffusion flame heights are drawn as a function of the nominal fuel vaporization Damköhler number, but without accounting for enhanced evaporation effects, i.e. all curves are plotted for $a_{c}=0$. These curves are plotted for several values of the initial water content of the water/fuel droplets in the spray, and will be used for comparative purposes when $a_{c} \neq 0$.

Two different sorts of flame heights are plotted. The solid lines represent the heights of a single diffusion flame, an example of which is drawn in Fig. 4. The broken line represents the height of a secondary flame that is established downstream for sufficiently low values of the vaporization Damköhler number. An example is illustrated in Fig. 5. 
The form of the curve for $\alpha=0$ in Fig. 3 was first presented in [11] for a fuel spray with no water content in the droplets. Its interpretation is as follows. It can be seen that for sufficiently low values of $\Delta_{F 0}$ insufficient fuel vapor becomes available to establish a significant flame so that a low flame is formed close to the fuel inlet and a secondary flame further downstream where more fuel vapor has been released. Although not illustrated here, in between the flame doublet, heterogeneous combustion of individual or clusters of droplets occurs. As the vaporization Damköhler number is increased to the region of the peak (in Fig. 3) a critical value is reached where a single homogeneous diffusion flame is established with most of the fuel supplied by droplets that evaporate upstream of it. As $\Delta_{F O} \rightarrow \infty$ all fuel droplets evaporate immediately at the chamber inlet so that the classical Burke-Schumann gaseous diffusion flame height is reclaimed.

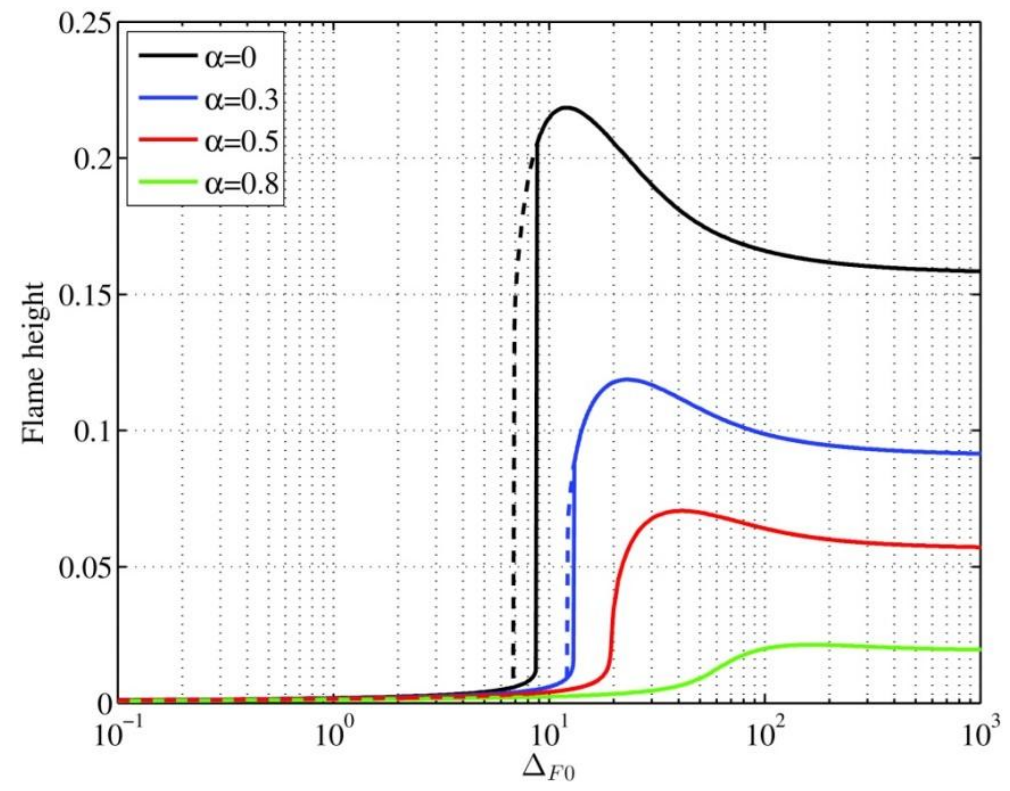

Figure 3: Diffusion flame height as a function of the nominal fuel vaporization Damköhler number for different values of the initial water content in the water/fuel sprays droplets; the curves are computed without accounting for enhanced evaporation, $a_{c}=0$. 


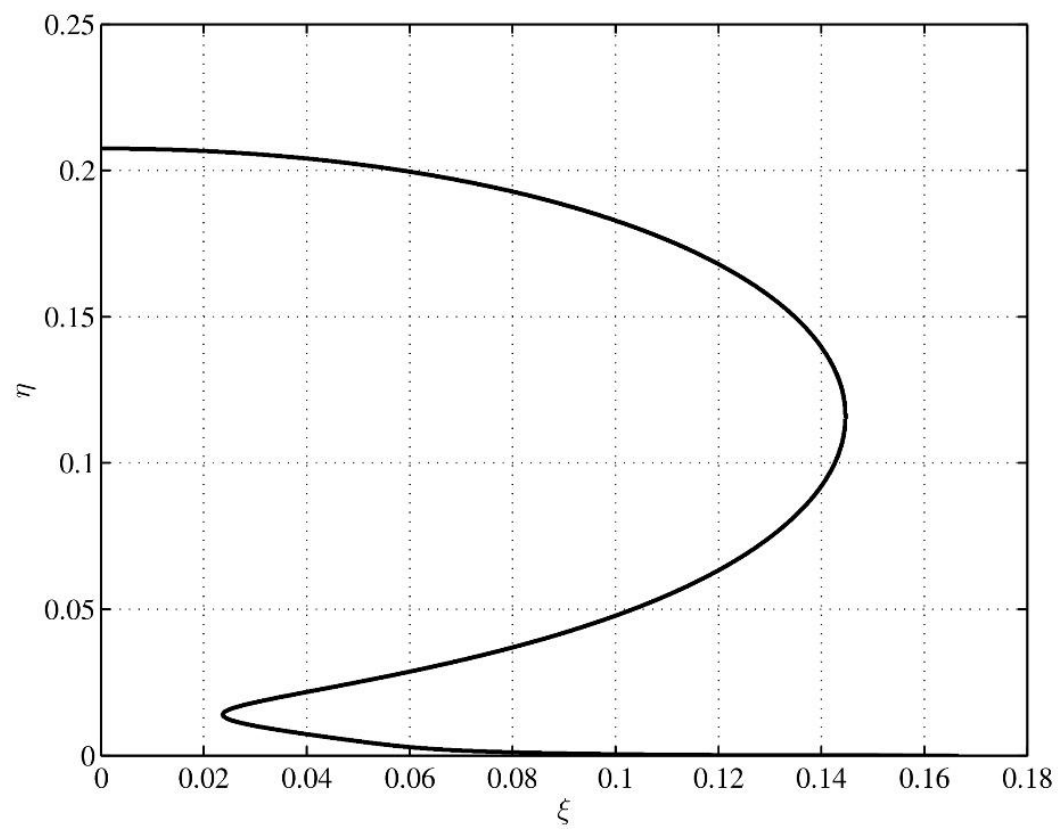

Figure 4: Example of the shape of a spray diffusion flame with pure fuel droplets; Data: $\Delta_{F 0}=9$.

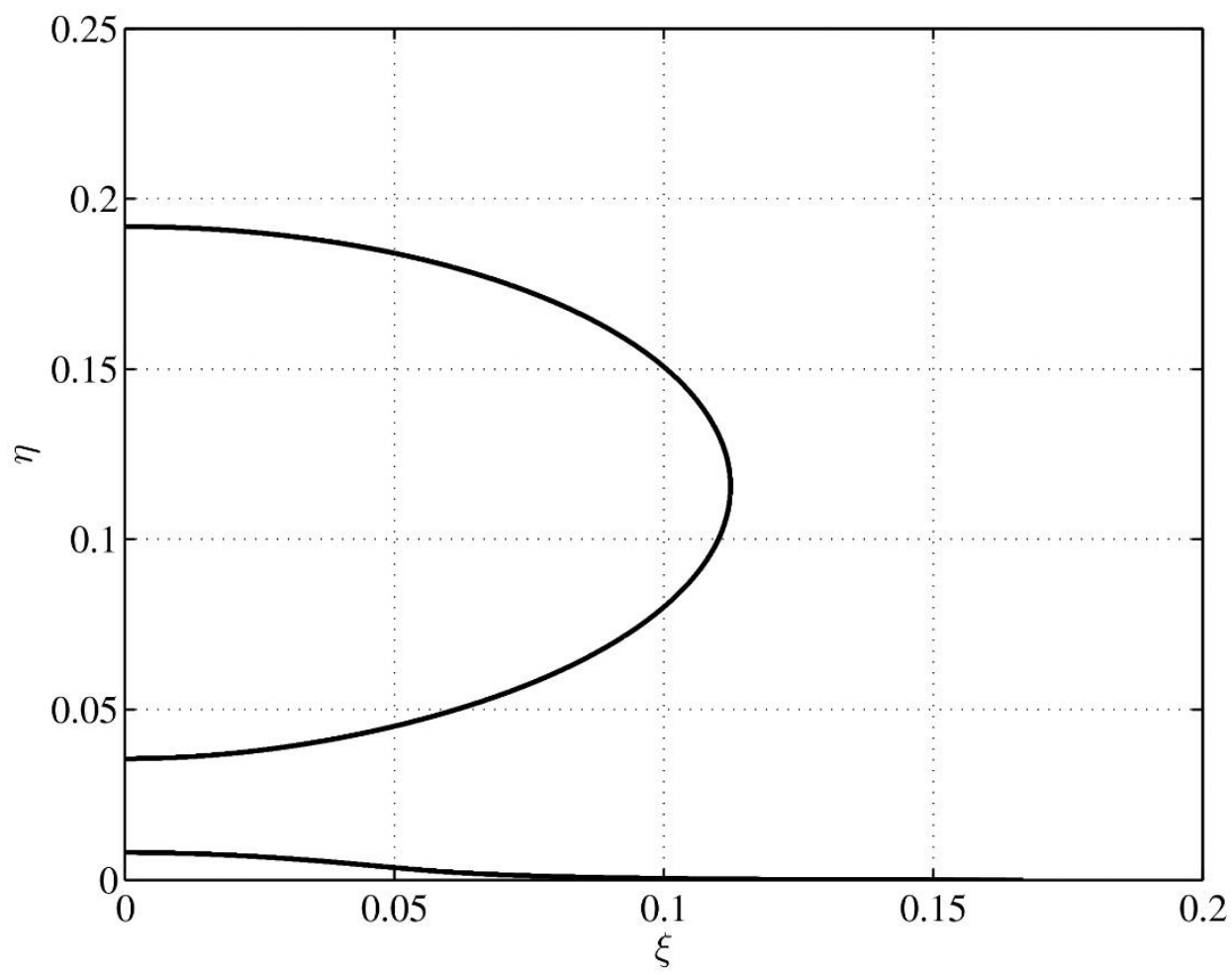

Figure 5: Example of the double spray diffusion flame structure with pure fuel droplets; Data: $\Delta_{F 0}=8$ 
Now consider in Fig. 3 the cases when there is water content in the fuel droplets, i.e. $\alpha \neq 0$. Since the total initial amount of liquid in the droplets is held constant less liquid fuel is available for evaporation and feeding the homogeneous diffusion flame. Thus, it is not surprising that the shapes of the curves for $\alpha \neq 0$ are similar to but lie below those when no water is present. For more than about $50 \%$ water content only low diffusion flames are formed with no secondary flames existing downstream. It is of interest to note how the maximum (normalized) temperature in these flames behaves as a function of the nominal vaporization Damköhler number for different water content in the sprays droplets. This is illustrated in Fig. 6. The behavior clearly mimics that of the flame height seen in Fig. 3 with any increase in the initial droplet water content leading to a decrease in the maximum temperature achieved.

In [3] appreciable improvement in fuel evaporation is reported for cases when $\alpha=0.8$ and it is this value that will be henceforth used.

Spray flame measurements are often given in terms of the number density of the spray's droplets. The current formulation is not given directly in terms of droplet number density. However, it can be shown that the parameter $\delta$ (see immediately after Eq. (20)) is proportional to number density. The dependence of the flame height on $\delta$, as a function of the nominal vaporization Danköhler number, when the water content is held constant using $\alpha=0.8$, is drawn in Fig. 7. The same qualitative behavior as shown in Fig. 3 is observed. However, the greater $\delta$ becomes the lower the flame height is due to the diminished fuel vapor that is initially available in the system. Conversely, as $\delta$ decreases the amount of fuel vapor initially available for 


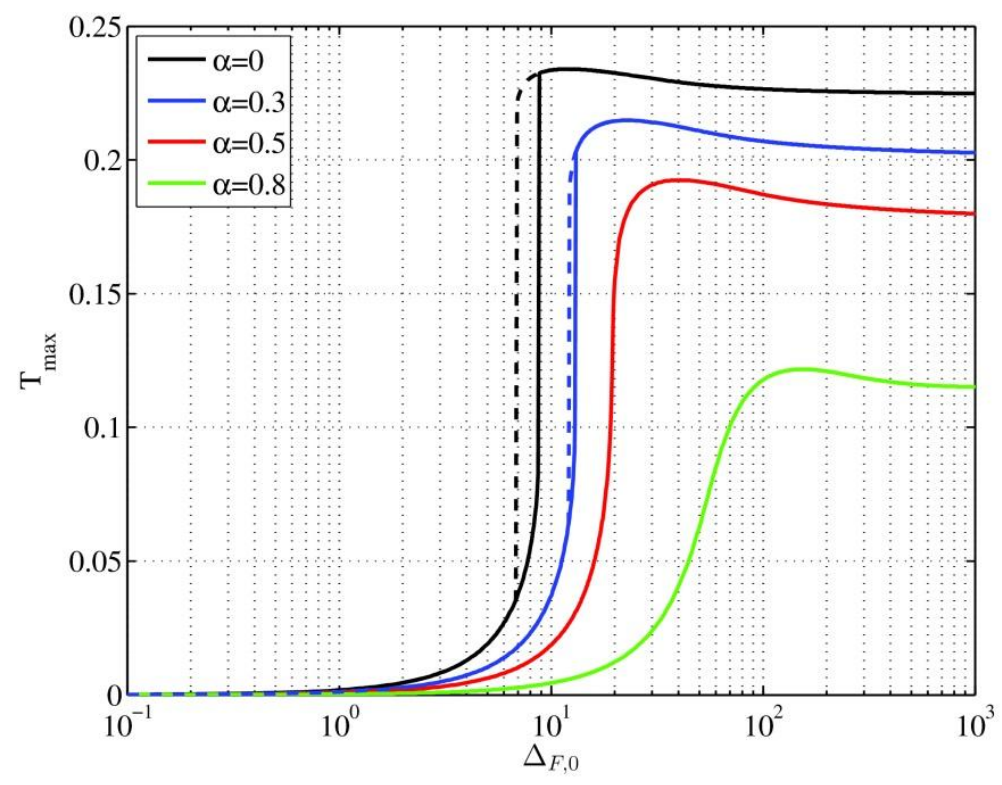

Figure 6: Maximum diffusion flame normalized temperature as a function of the nominal fuel vaporization Damköhler number for different values of the initial water content in the water/fuel sprays droplets; the curves are computed without accounting for enhanced evaporation, $a_{c}=0$.

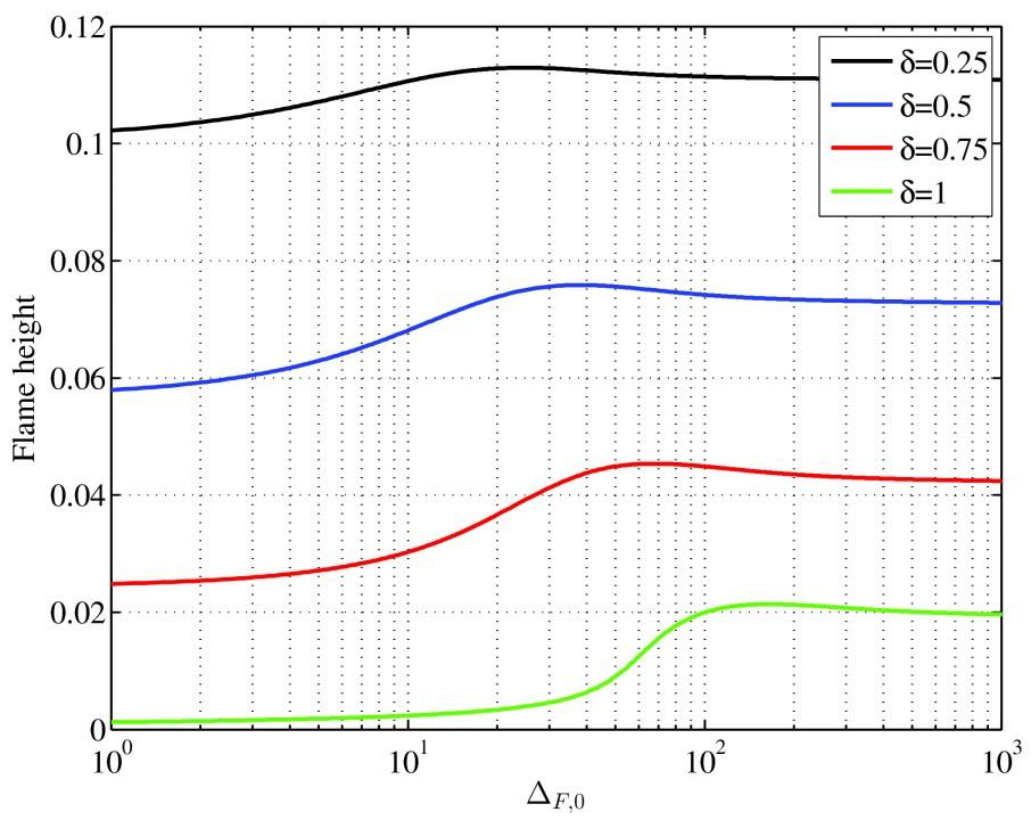

Figure 7: Diffusion flame height as a function of the nominal fuel vaporization Damköhler number for different values of $\delta$ (the initial fraction of liquid fuel and water in the total fuel/water supply (which may also include fuel vapor)); the curves are computed without accounting for enhanced evaporation, $a_{c}=0$ and for $\alpha=0.8$. 
combustion increases (see boundary condition Eq. (18)) thereby enabling a greater flame height.

Attention is now turned to the water/fuel (t-butanol) spray with enhanced evaporation (i.e. $a_{c} \neq 0$ ). As is evident from Eq. (21) the fuel vaporization Damköhler number will evolve downstream, as a function of $\eta$. Part of this $\eta$-dependence is due to the evaporation of the water content of the droplets and is therefore dependent on the (constant) water vaporization Damköhler number, $\Delta_{w}$. For the sake of generality the extent to which this parameter impacts on the process can be artificially assessed as follows. In Fig. 8 the downstream development of the fuel vaporization Damköhler number's dependence on the water vaporization Damköhler number is drawn. It can be seen that as the water vaporization Damköhler number decreases it impacts more strongly on $\Delta_{F}$ thereby providing significant enhanced evaporation of liquid fuel. For values of $\Delta_{W}$ up to about $10 \%$ of the value of $\Delta_{F}$ at $\eta=O$ the impact is strongest and it is for this reason that the value of $\Delta_{F O} / \Delta_{W}=100$ was taken for the calculations. Note also that, although the nominal vaporization Damköhler number for the pure fuel is 100 , the initial water content in the sprays droplets implies an increase to 112 at $\eta=0$. In Fig. 9 the flame shapes corresponding to the underlying vaporization Damköhler number behavior of Fig. 8 are shown. Note the way the flame shapes close to $\eta=0$ turn sharply left into the region above the inner duct but then turn outward as the fuel vaporization Damköhler number increases and fuel vapor is more rapidly supplied to fuel the homogeneous flame front. The flame shapes are similar to each other, but as the water vaporization Damköhler number artificially increases the flames become narrower and their heights decrease. This is as anticipated from Fig. 3. 


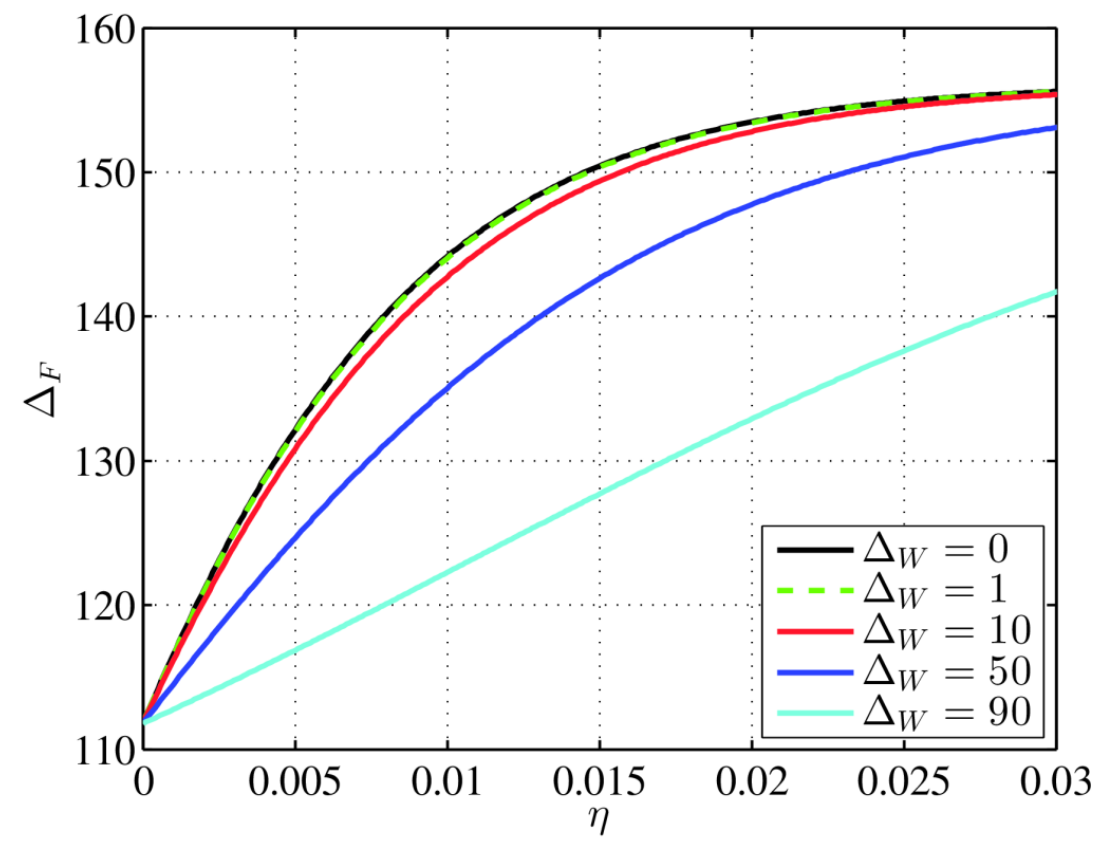

Figure 8: Downstream evolution of the influence of the water vaporization Damkohler number on the fuel vaporization Damköhler; Data: $\Delta_{F, 0}=100$.

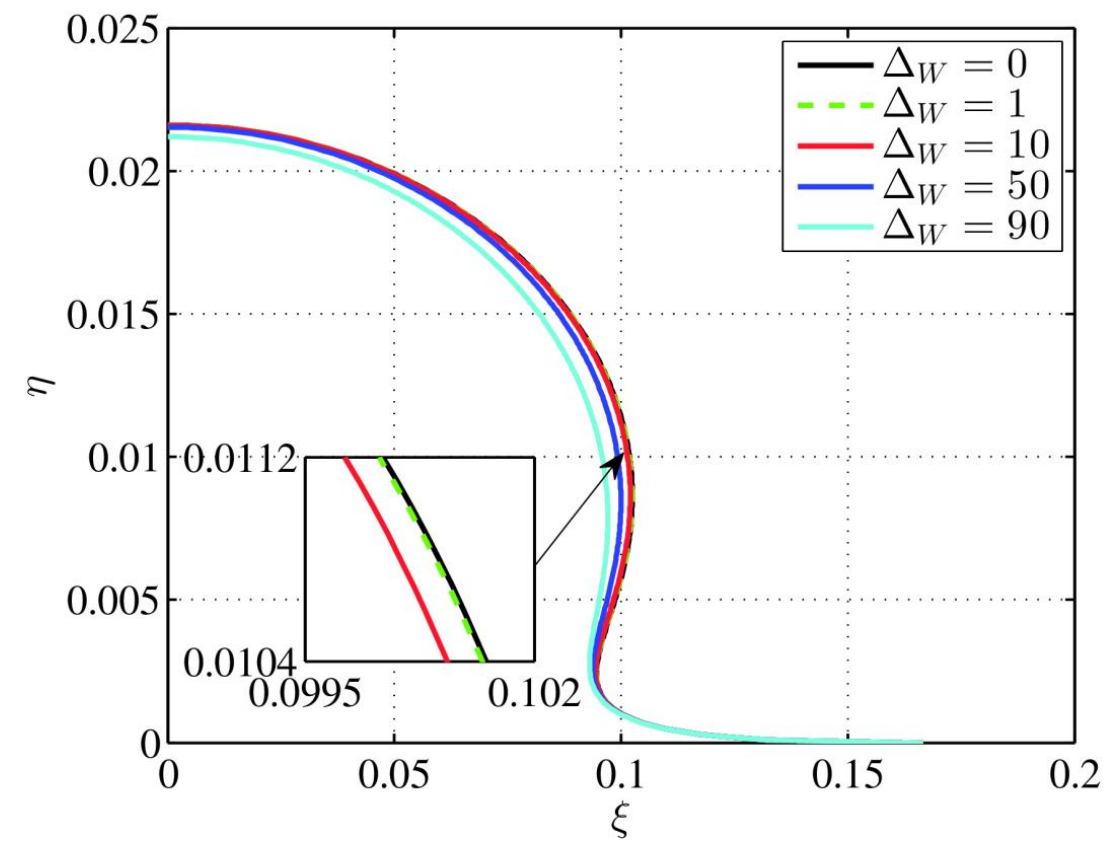

Figure 9: Spray diffusion flame shape dependence on water vaporization Damköhler number; Data: $\Delta_{F, 0}=100$.

We now compare two specific cases of water/fuel sprays, one in which the droplets are comprised of water and t-butanol and the other having water and ethylene glycol. These two are chosen based on Fig. 2 with the t-butanol having the largest range of 
activity coefficient and the ethylene glycol having the smallest range amongst all chemicals considered in [3]. In Fig. 10 flame heights are plotted for these two cases as a function of the nominal fuel vaporization Damköhler number. The behavior of the diffusion flame height is similar to that found in Fig. 3. For the water-ethylene glycol combination the curve is almost indistinguishable from that of the ideal solution (i.e. $\left.a_{c}=0\right)$. The water+t-butanol spray flames are all higher due to the stronger preferential fuel evaporation and the effect of the normalized activity coefficient leading to a supply of vapor earlier upstream. The highest water+t-butanol flame is therefore achieved for a lower value of the nominal fuel vaporization Damköhler number than for the water+ethylene glycol case.

It is instructive to compare the flame shapes (see Fig. 11) for the maximum flame heights obtained in Fig. 10. Before doing so the significance of the nominal vaporization Damköhler number is reiterated. This general spray-related number captures within it factors concerning the evaporation rate of the fuel's component(s), droplet sizes in the spray, inter-droplet spacing in the spray, the thermal state of the spray's droplets and other information stemming from how an individual droplets behavior within a spray context is modeled (see, [8],[15],[20],[21]). Thus, two quite different sprays can have the same nominal vaporization Damköhler number depending on the different roles of the aforementioned factors for each spray. For example, drastically different internal droplet size distribution and droplet proximity within two sprays of different fuels having dissimilar evaporation rates can result in the same nominal vaporization Damköhler number for each spray.

Now, returning to Fig. 10, when $a_{c}=0$ the peak flame height occurs when the nominal vaporization Damköhler number is $\Delta_{F, 0}=165$. When enhanced evaporation 
occurs the peak height shifts to the left at $\Delta_{F, 0}=162$ for the water-ethylene spray flame and further to the left at $\Delta_{F, 0}=112$ for the water-t-butanol spray flame. Despite these shifts the flame shapes bear a similarity to each other. Note that there is little difference between the water-ethylene glycol flame (for which the enhanced evaporation is weak) and the flame for which there is no enhanced evaporation (the ideal solution). However, for the water-t-butanol spray flame there are some differences but these are minor since the relevant nominal vaporization Damköhler numbers are close to one another. The base of the t-butanol flame is narrower due to the lower nominal vaporization Damköhler number, which implies a slower rate of fuel evaporation. Further downstream the effect is reversed as the actual rate of evaporation increases due to the high enhanced evaporation of t-butanol. The peak of the water-t-butanol spray flame is fractionally higher than that of the other flames shown. In any event it is important to notice that the flames are short due to the high water content of the spray's droplets.

However, if two completely identical sprays are considered with the only difference between them being the material components of the droplets, then a comparison between the t-butanol/water and ethylene glycol/water spray flames roughly reflects their respective evaporation rates. Since the ratio of the evaporation rate of t-butanol to that of ethylene glycol is of the order of 10, the flame shapes are quite distinct (see Figure 12). 


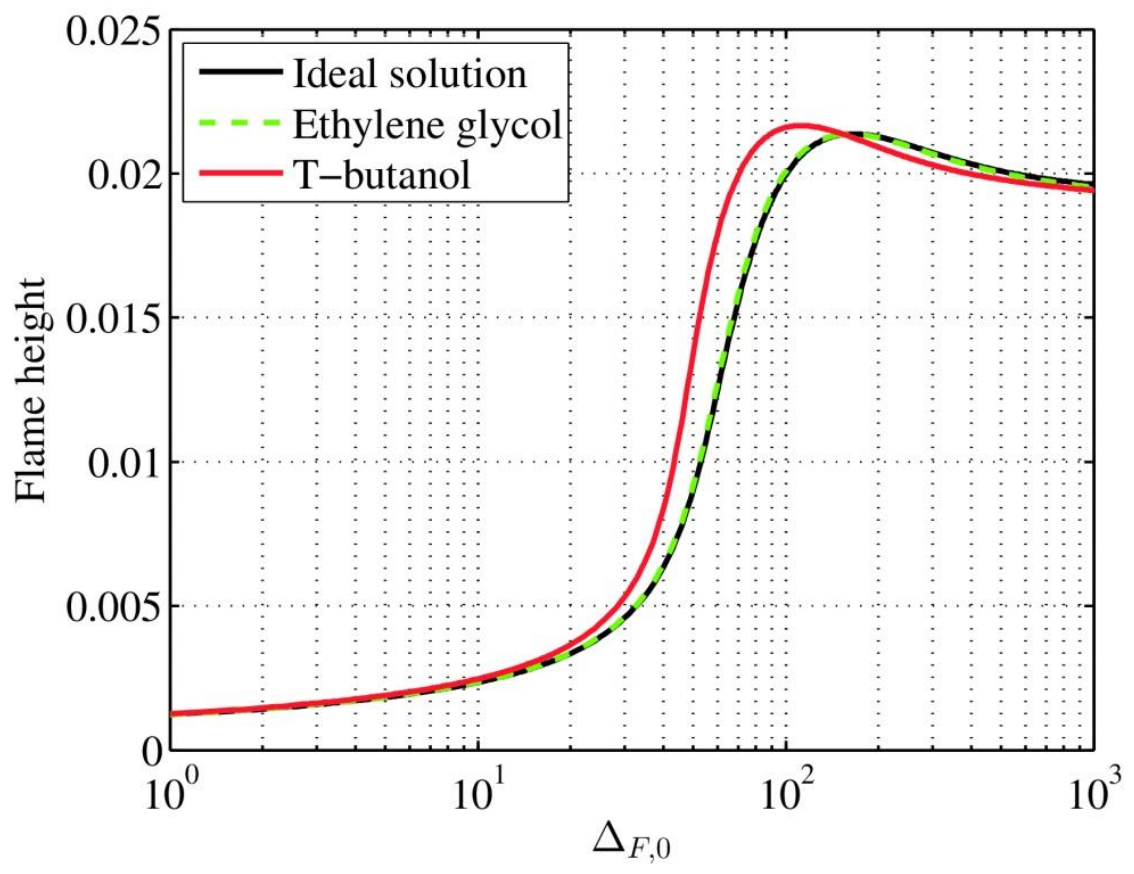

Figure 10: Diffusion flame height as a function of the nominal fuel vaporization Damköhler number for water+t-butanol and water+ethylene glycol sprays; the curves are computed accounting for enhanced evaporation, $a_{c} \neq 0$, the black line is for the case with no enhanced evaporation.

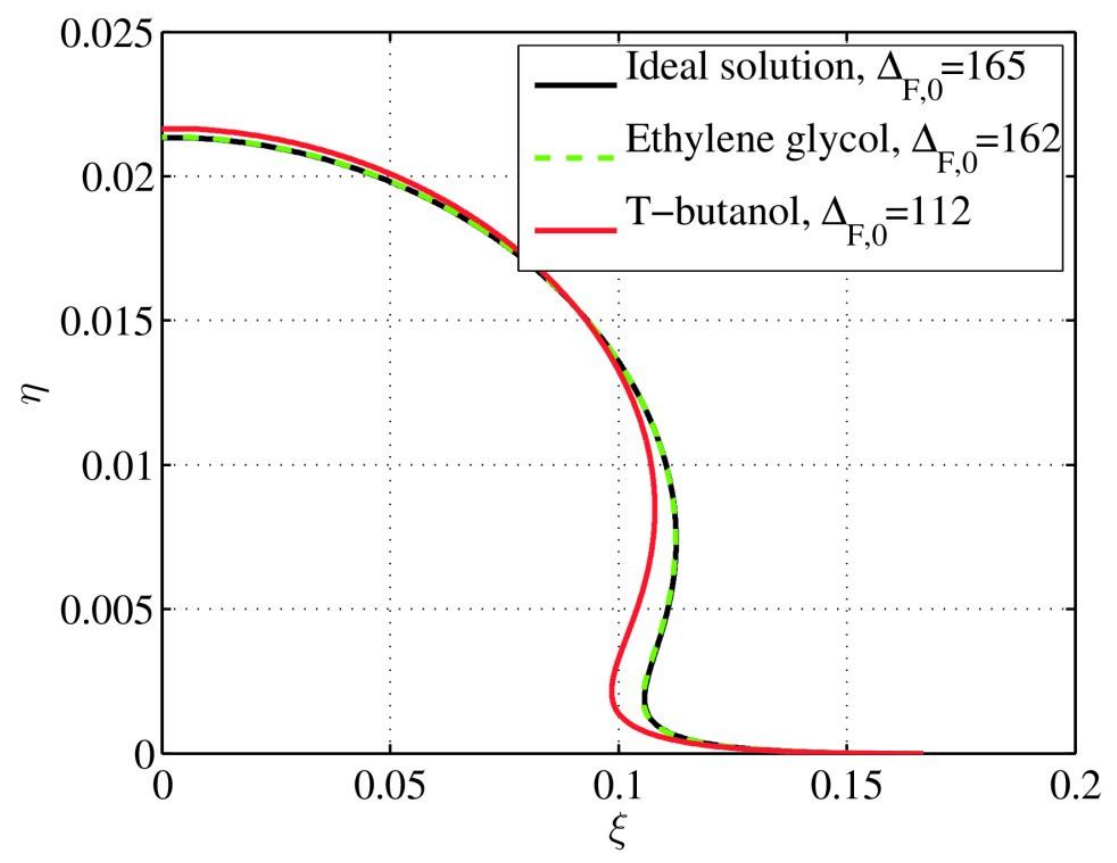

Figure 11: Shapes of highest diffusion flames for water+t-butanol and water+ethylene glycol spray flames; the curves are computed accounting for enhanced evaporation, $a_{c} \neq 0$, the black line is for the case with no enhanced evaporation. 

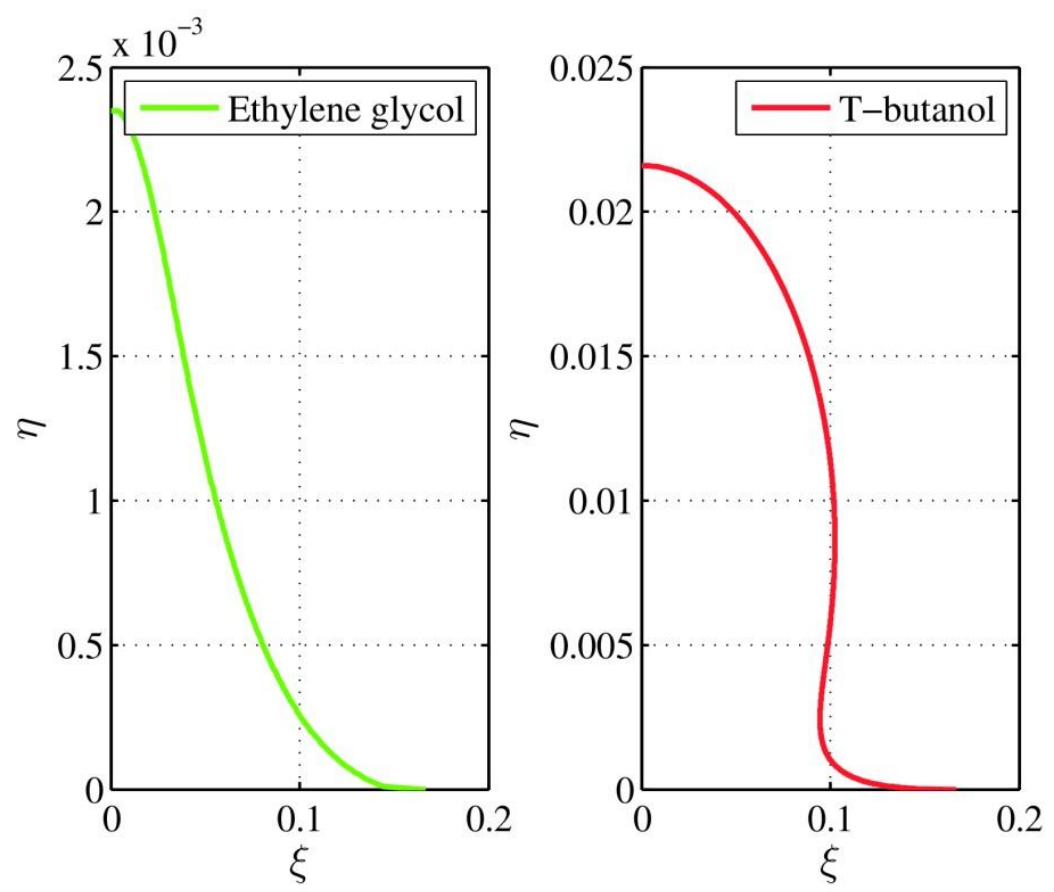

Figure 12: Flame shapes for water+ethylene glycol and water+t-butanol spray flames having otherwise identical sprays; the curves are computed accounting for enhanced evaporation, $a_{c} \neq 0, \Delta_{F 0}$ for t-butanol 100, $\Delta_{F 0}$ for ethylene glycol 10.

The influence of the oxygen in the outer ducts on water/fuel spray flames is now examined. In this simplified model it is the parameter $V$ that describes the oxygen normalized mass fraction. Until now use has been made of a value $V=0.306$. In Fig. 13 a comparison is made between flame heights as a function of nominal fuel vaporization Damköhler number for three values of $V(0.2,0.306$ and 0.5$)$ for the two cases of water+t-butanol and water+ethylene glycol spray diffusion flames, and the case where enhanced fuel evaporation is artificially suppressed (the ideal solution). 


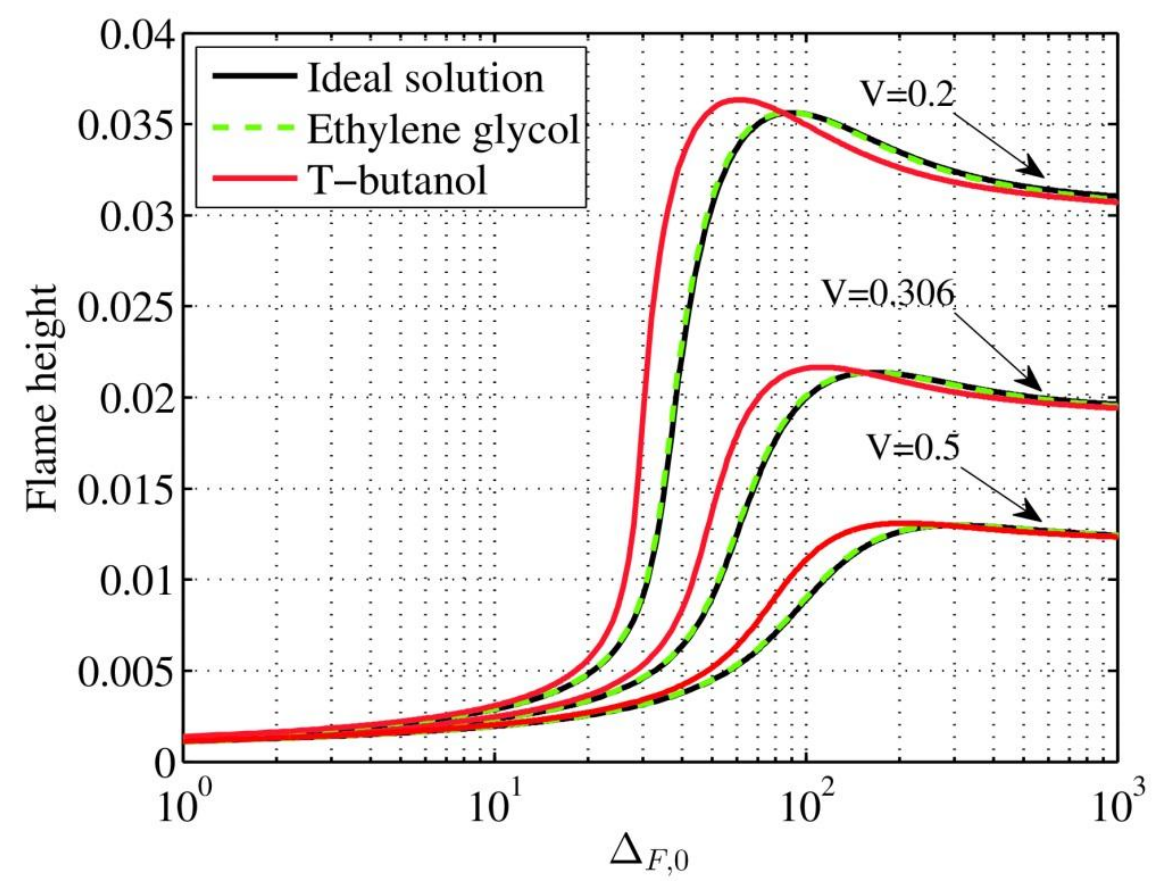

Figure 13: Influence of oxygen supply conditions on diffusion flame height as a function of the nominal fuel vaporization Damköhler number for water+tbutanol and water+ethylene glycol sprays; the curves are computed accounting for enhanced evaporation, $a_{c} \neq 0$, the black line is for the case with no enhanced evaporation.

It is well known that in a Burke-Schumann configuration a reduction in the supply oxygen mass fraction leads to increased flame height [22]. In Fig. 13 this is clearly the case for the all spray flames considered here with factors of as much as four being encountered for certain values of $\Delta_{F, 0}$. Apart from the flame heights the general trend is unchanged from that discussed in connection with Fig. 10.

In Fig. 14 the flame shapes are shown corresponding to $\Delta_{F, 0}=95$. Once again the enhanced vaporization of the water-t-butanol spray flame is evident for all oxygen supply conditions. In addition, the inverted $\mathrm{S}$-shape of the flames for $V=0.2$ is more pronounced particularly for the water-t-butanol spray flame. However, whereas the height of this flame is greater than the heights of the water-ethylene glycol and ideal 
flames when $V=0.306$ and $V=0.5$ by about $1 \%$, the other two flames are slightly higher when $V=0.2$.

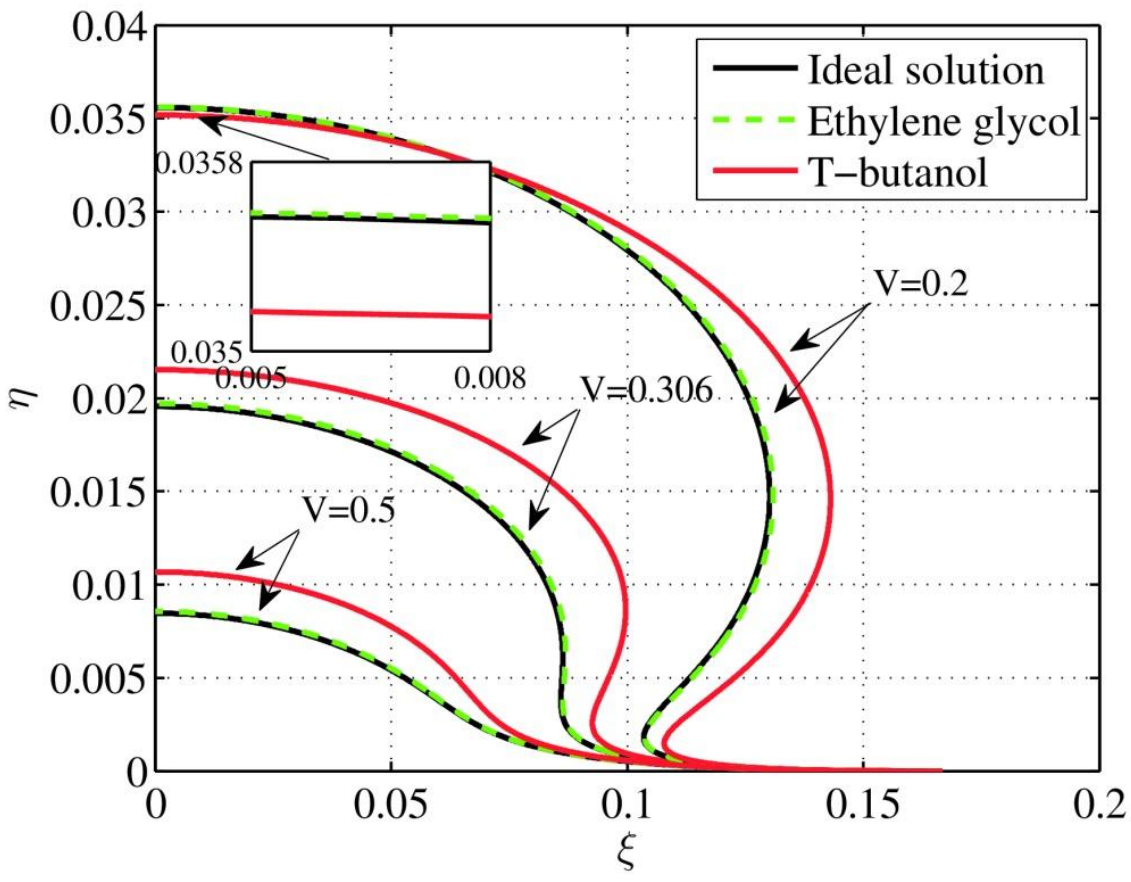

Figure 14: Shapes of highest diffusion flames for water+t-butanol and water+ethylene glycol spray flames; the curves are computed accounting for enhanced evaporation, $a_{c} \neq 0$, the black line is for the case with no enhanced evaporation; Data: $\Delta_{F, 0}=95$.

The normalized temperature profiles along the flame fronts are considered in Fig. 15. The abscissa, $s$, is measured from the base of the flame. Because they are more energetic the flames formed with more oxygen supplied attained greater temperatures than those with a lower oxygen supply. However, of particular interest is the higher temperature attained by the water-t-butanol flame when $V=0.306$ and $V=0.5$ as compared to the other two cases considered here. This is a striking indication of the thermal influence of enhanced evaporation. Nevertheless, when $V=0.2$ the trend is reversed at the upper part of the flame. At this location on the flame height versus nominal vaporization Damkohler number graph, Fig. 13, the water-t-butanol flame 
has passed the peak flame height value and is characterized by droplet evaporation entirely upstream of the flame front. As a result, heat loss due to evaporation is more focused in the pre-flame region thereby impacting more strongly on the thermal field.

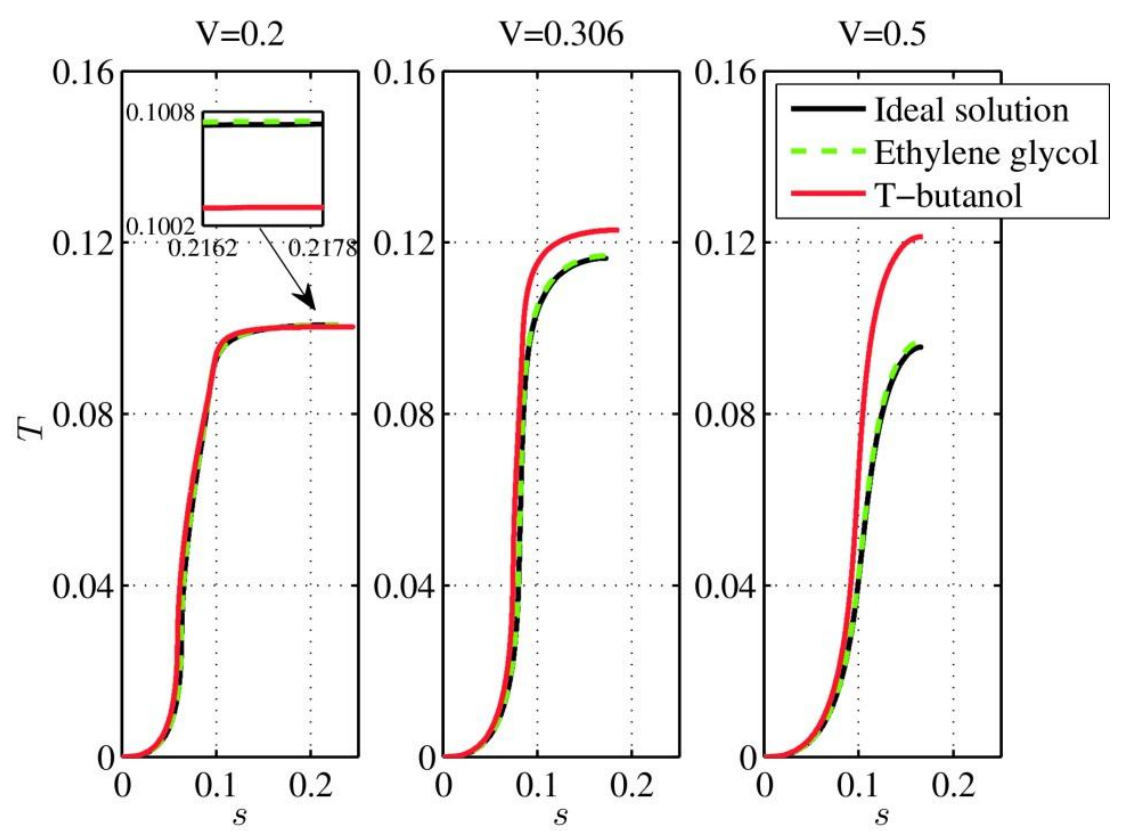

Figure 15: Normalized temperature profiles along diffusion flames of water $+t-$ butanol and water+ethylene glycol sprays; the curves are computed accounting for enhanced evaporation, $a_{c} \neq 0$, the black line is for the case with no enhanced evaporation; Data: $\Delta_{F, 0}=95, \boldsymbol{s}$ is measured from the flame base.

In contrast, the water-ethylene glycol spray flame has a pre-peak flame height for $\Delta_{F, 0}=95$ so that droplet evaporation (and burning) is also occurring downstream of the homogeneous diffusion flame front. In this case the heat loss is less focused thereby leading to a slightly higher flame temperature. 


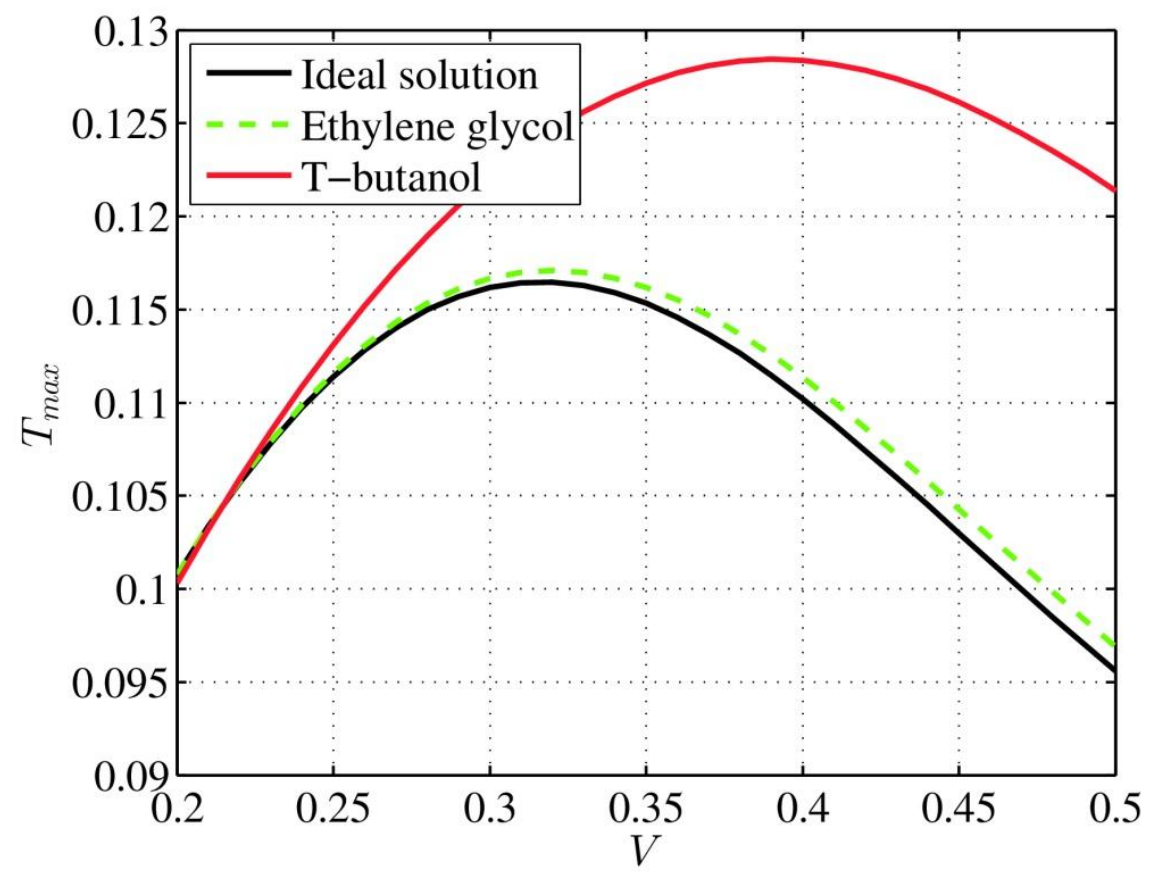

Figure 16: Maximum flame temperature as a function of oxygen supply conditions for diffusion flames of water+t-butanol and water+ethylene glycol sprays; the curves are computed accounting for enhanced evaporation, $a_{c} \neq 0$, the black line is for the case with no enhanced evaporation; Data: $\Delta_{F, 0}=95$.

Finally, In Fig. 16 the maximum temperature is plotted as a function of the oxygen supply conditions for the diffusion flames of Fig. 15 when $\Delta_{F, 0}=95$. The higher temperature that can be strikingly achieved through the combination of both a higher oxygen supply condition and enhanced evaporation of the t-butanol+water mixture is rather evident.

The current analysis was carried out under the assumption of infinite chemical Damköhler number so that the way in which the afore-described thermal effects influence flame extinguishment are beyond the scope of this work. Nevertheless, the results do underscore the delicate way in which combined usage of the oxygen supply along with enhanced evaporation effects can, under appropriate conditions, produce more rigorous combustion exhibited by higher flame temperatures thereby being more 
inclined to maintain flame stability, in spite of the high percentage of water in the sprays droplets.

\section{Conclusions}

The classical Burke-Schumann diffusion flame was adopted as a paradigm configuration for examining spray diffusion flames in which the spray's droplets are comprised of a mixture of water and fuel. Building on experimental data and simple modeling predictions from the literature, which testify to the viability of burning fuels heavily diluted with water, a special fuel vaporization Damköhler number was constructed to reflect both preferential fuel evaporation and non-ideal solution behavior of the spray's droplets. An analytical solution of the governing equations was found. Calculations based on the solution enabled a comparison between water-tbutanol and water-ethylene glycol spray diffusion flames. The former spray was characterized by strong enhanced fuel evaporation whereas the latter by weak enhanced evaporation. This leads to differences in flame shapes, heights and temperatures. It was argued that there exists the possibility of sustaining water-fuel spray diffusion flames by combining the enhanced fuel evaporation and control of the oxygen supply.

The main restriction of the current model is the use of an infinite chemical Damköhler number so that the flame collapses to an infinitesimally thin flame front. More realistic finite rate chemistry will enable deeper insight into questions of stability, extinction and unsteadiness of these water/fuel spray flames. In addition, realistic sprays are multisize. Ongoing research is currently addressing these critical questions. 


\section{Acknowledgements}

JBG acknowledges the partial support of the Lady Davis Chair in Aerospace Engineering. RLA acknowledges the support of the National Science Foundation (1337008) and the Consortium for Clean Coal Utilization.

\section{References}

[1] F. Yi, " Oxy-combustion of High Water Content Fuels", PhD thesis, School of Engineering \& Applied Science, Department of Energy, Environmental \& Chemical Engineering, Washington University in St.. Louis, St Louis, Missouri, USA, 2013.

[2] F. Yi and R.L. Axelbaum, "Stability of spray combustion for water/alcohols mixtures in oxygen-enriched air", Proceedings of the Combustion Institute, Vol. 34, pp. 16971704, 2013.

[3] F. Yi and R.L. Axelbaum, "Utilizing preferential vaporization to enhance the stability of spray combustion for high water content fuels", Combustion and Flame, Vol. 161, pp. 2008-2014, 2014.

[4] http://www.cleanenergysystems.com/ (Last accessed on 20.05.15).

[5] F.R. Newbold and N.R. Amunson, "A model for evaporation of a multicomponent droplet", AIChE Journal, Vol.19 (1), pp. 22-30, 1973.

[6] C.K. Law, "Internal boiling and superheating in vaporizing multicomponent droplets", AIChE Journal, Vol.24 (4), pp. 626-632, 1978.

[7] H. Zhang and C.K. Law, "Effects of temporally varying liquid-phase mass diffusivity in multicomponent droplet gasification", Combustion and Flame, Vol. 153, pp. 593-602, 2008.

[8] S. Sazhin, "Droplets and Sprays", Springer, 2014. 
[9] S.-S. Hou, J.-C. Lin and C.-Y. Hsuan, "The interaction between internal heat gain and heat loss on compound-drop spray flames", International Journal of Heat and Mass Transfer, Vol. 71, pp. 503-514, 2014.

[10] S. P. Burke and T. E. W. Schumann, "Diffusion flames", Indust.Eng. Chem., Vol. 20 (10), pp. 998-1004, 1928.

[11] J. B. Greenberg, "The Burke-Schumann flame revisited - with fuel spray injection," Combustion and Flame, Vol. 77, Nos. 3 \& 4, pp. 229-240, 1989.

[12] J.B. Greenberg, "Spray diffusion flames with arbitrary initial droplet velocity distributions", Combustion Science and Technology, Vol. 75, pp. 13-30, 1991.

[13] J. Arrieta-Sanagustin, A.L. Sanchez, A. Linan and F.A. Williams, "Couplingfunction formulation for monodisperse spray diffusion flames with infinitely fast chemistry", Fuel Processing Technology, Vol. 107, pp. 81-92, 2013.

[14] A.L. Sanchez, J. Urzay and A. Linan,"The role of separation of scales in the description of spray combustion", Proceedings of the Combustion Institute, Vol. 35, pp. 1549-1577, 2015.

[15] J.B. Greenberg, I. Silverman and Y. Tambour, "On the origins of the spray sectional conservation equations", Combustion and Flame, Vol. 93, 90-96, 1993.

[16] J. B. Greenberg, A.C. McIntosh and J. Brindley, "Linear stability analysis of laminar premixed spray flames", Proceedings of the Royal Society of London, Series A: Mathematical, Physical and Engineering Sciences, Vol. 457, No.2005, pp.1-31, 2001.

[17] N.A. Chigier, Energy, Combustion and Environment,McGraw-Hill, 1981.

[18] W.O.H. Mayer and J.J. Smith, "Fundamentals of supercritical mixing and combustion of cryogenic propellants", in Liquid Rocket Thrust Chambers: Aspects of Modeling, Analysis, and Design edited by V. Yang, M. Habiballah, J. Hulka and M. 
Propp, Progress in Astronautics and Aeronautics, Vol. 200, American Institute of Aeronautics and Astronautics,Virginia, USA, 2004.

[19] S. Russo and A. Gomez, "Physical characterization of laminar spray flames in the pressure range 0.1-0.9 MPa", Combustion and Flame, Vol. 145, pp. 339-356, 2006.

[20] K. Annamalai and W. Ryan, "Interactive processes in gasification and combustion, Part 1: Liquid drop arrays and clouds", Progress in Energy and Combustion Science, Vol. 18, pp. 221-295, 1992.

[21] T. Elperin and B. Krasovitov, "Analysis of evaporation and combustion of random clusters of droplets by a modified method of expansion into irreducible multipoles", Atomization and Sprays, Vol. 4(1), pp. 79-97, 1994.

[22] S.H. Chung and C.K. Law, "Burke-Schumann with streamwise and preferential diffusion", Combustion Science and Technology, Vol. 37 (1-2), pp. 21-46, 1984. 